\title{
Complex structures in cavities with media displaying EIT: coexistence, defects and selection mechanism
}

\author{
M. Eslami \\ Department of Physics, University of Guilan, P.O. Box 41335-19141 Rasht, Iran \\ E-mail: meslami@guilan.ac.ir
}

\section{R. Kheradmand}

Research Institute for Applied Physics and Astronomy, University of Tabriz, Tabriz, Iran

\section{G.-L. Oppo}

Department of Physics, University of Strathclyde, Glasgow, G4 0NG, Scotland, EU

June 2019

\begin{abstract}
A cavity filled with a medium displaying electromagnetically induced transparency is shown to exhibit coexistence of complex transverse structures often leading to pattern competition. Because of multi-stability of the solutions, the asymptotic state in such a cavity crucially depends on the values of the control parameters and the initial conditions. The pattern competition can result in coexisting regions of pattern structures of different geometry separated by stable or metastable fronts. Here we propose a selection technique based on external periodic modulations for directing the system to a single pattern state of choice and thus removing the separation fronts. The control technique takes the system close to sub-sections in the phase space providing the operator with the choice of the final state from different multi-stable solutions. We extend the use of the technique to stationary or drifting structures composed of regions made of a single pattern state with different orientations. In this case the regularization to a pattern with a single orientation is associated with the removal of defects. The harmonic signal technique has not been used previously to control a spatio-temporal system where the multi-stability is due to the coupling of nonlinearity and diffraction.
\end{abstract}

\section{Introduction}

Electromagnetically induced transparency (EIT) is an example of a coherent multilevel process and has opened a promising window for realization of schemes needed for quantum information systems, coherent control of atomic populations, and mediation of interactions between optical fields [1]. The majority of EIT studies have been carried out 
in gaseous media where the dominant broadening mechanism is that of a homogenous type leading to a variety of applications including slow light propagation [2,3], optical storage [4], precision measurements [5], amplification [6] and lasing without inversion [7]. More recently, EIT has also been reported in solid state media featuring inhomogeneous line-shape broadening which helps in the implementation of scalable and integratable quantum optical and photonic devices $[8,9,10]$.

Complex spatial structures in the light intensity output of an optical cavity containing a medium close to EIT have shapes and stability with strong dependence on parameter values and initial conditions [11]. Sensitivity to initial conditions in the final evolution of this system is due to a generalized multi-stability. The multi-stability and the nature of the stable states is in turn affected by relatively small changes in the parameter values since different branches of solutions experience different sequences of bifurcations. For some initial conditions, a given set of expected spatially periodic solutions is attained. For other initial conditions, however, the spatio-temporal evolution moves the system in different directions in the phase space to either coexistent regions of different patterns or to stable defects surrounded by regions of different orientation of a single transverse pattern $[11,12]$. In the first case, contiguous or nested patterns are separated from each other by stable fronts [13]. This sort of complexity in nonlinear optics is due to large coherence of the light-matter interaction and has recently been shown to trigger extreme events in the transverse section of a cavity with triple quantum dot molecules where tunneling induced transparency (TIT) replaces EIT [14].

Multi-stability of stationary or oscillating states is a nonlinear phenomenon that occurs in many branches of science and technology. It has been extensively discussed in lasers $[15,16]$, brain activity [17], chemical oscillations [18], and even climate change [19]. These examples focus on systems described by sets of nonlinear ordinary differential equations but, of course, multi-stability becomes even more commonplace in timedelayed [20], networked [21] and spatio-temporal [22] systems because of their intrinsic high dimensionality. In nonlinear optics, spatio-temporal multi-stability has been described in laser [23], liquid crystal [24] and photorefractive [25] devices.

The high sensitivity to initial conditions in determining the final state of multi-stable systems bears some similarity to chaotic systems that evolve in a phase space that is dense in unstable periodic orbits [26]. In our case, the dynamics in different regions of the transverse plane meanders among several stable and unstable patterns of different shape, size and orientation. In this respect the transient dynamics has similarities with chaotic systems. The different spatial regions of the transverse plane are, however, coupled to neighboring regions via diffraction so that either one stable pattern with a single orientation dominates the final state or regions of different patterns or same pattern with different orientations survive asymptotically via the stabilization of fronts or defects. The presence of boundary conditions can slow or accelerate the dynamics of the patten domains to reach the final state. The number of nested domains or domains of different orientation increases the larger is the ratio between the system size and the pattern wavelength. 
In several physical systems, behaviors originating from a chaotic dynamics can be controlled by applying appropriately designed small perturbations to one of the control parameters to stabilize a desired periodic behavior rather than a chaotic one. Generally speaking, unstable steady states (USSs) and unstable periodic orbits (UPOs) that are embedded in the chaotic attractor characterizing the dynamics in phase space are at the base of most schemes for the control of chaos [27]. After the pioneering work of Ott et al. [28], it has been the common practice of the majority of chaos-control protocols in optics to stabilize one such UPO by introducing small adjustments to the control parameter of the system when the current state of the system is in the vicinity of the targeted UPO [29]. In the case of spatially extended systems, similar goals have been achieved by small spatial modulation to the input pump field derived from the Fourier transform of the output electric field $[30,31]$ or by target patterns applied using an interfaced computer through a feedback technique [32] .

The nonlinear optical configuration studied here deals with a generalized multi-stability with two or many more stable solutions (with different orientation) simultaneously present in the system. In such a situation, the system may (or may not) display a final state with one single stable pattern with a single orientation depending on the chosen initial condition. In the purely temporal case, including delayed systems, several nonfeedback methods to control multi-stability have been introduced, namely, pulse control, where short pulses are used to select particular attractors in a multi-stable system and parametric forcing leading to annihilation of attractors and thereby turning the multi-stable system to a mono-stable one [33, 34]. Here we apply similar techniques to a model of a cavity filled with a medium displaying EIT, i.e. a spatio-temporal system where the multi-stability is due to optical nonlinearity and light diffraction. Analogously to chaotic systems, we achieve a transition to targeted solutions by the use of a wise choice of a temporally modulated perturbation that can direct the trajectory towards a specific attractor and produce a series of desired states. We chose one of the stable solutions that the system can display as a target. The control signal is capable to move the system from one stable state to another one with good advantages for the operator. We show that by applying appropriately designed harmonic perturbations one can target one of the multi-stable solutions in situations where growth from noise generates either stable regions of different pattern geometries or of different orientation. To the best of our knowledge, this is the first study where control of multi-stability through a harmonic signal technique has been achieved in a spatio-temporal system described by partial differential equations.

The paper is organized as follows: in Sec. II we recall the model of an EIT medium in a cavity pumped by two input laser beams and its multi-stable features [11]. In particular we highlight the role of the control parameters and initial conditions. In Sec. III, we discuss the feasibility of the harmonic perturbation technique to decompose a spatial structure formed by stable regions of coexisting patterns into its building elements. Removal of defects from disordered patterns has been achieved by using a control signal from an all-optical feedback loop with a spatial Fourier filtering [35]. Here instead we 


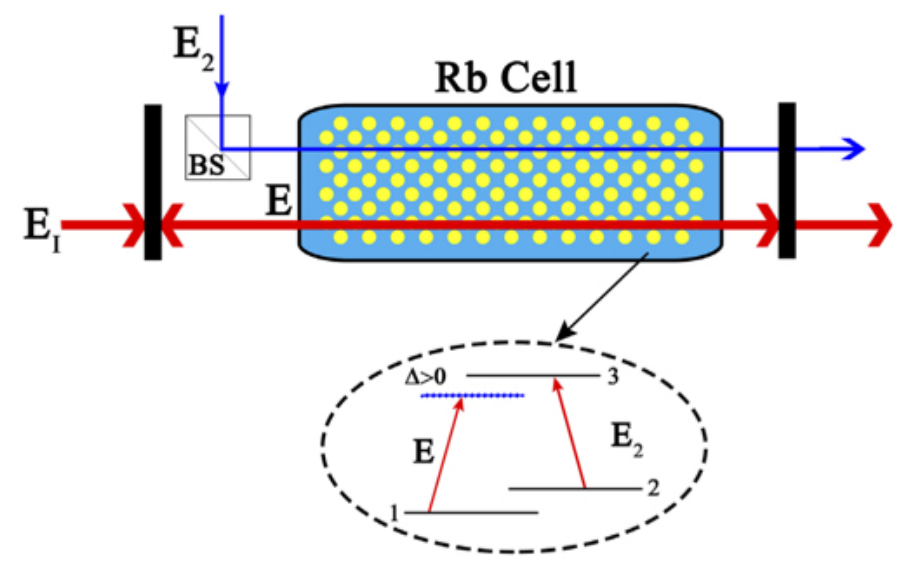

Figure 1. (Color online) Configuration of the employed model and the 3-level $\Lambda$ type atomic system with self-focusing nonlinearity.

show that a harmonic perturbation with appropriate amplitude, phase and duration applied to the case of stable regions of a pattern with different orientations can also produce a single pattern state by removing its defects in Sec. IV. In Sec. V we consider a finite input pump to check the robustness of the proposed method in situations closer to experimental realizations. Concluding remarks appear in the final Sec. VI.

\section{The model}

The system is composed by a $\Lambda$ three level medium inside a cavity and pumped by two input fields $E_{I}$ and $E_{2}$ of frequency close to the two atomic resonances respectively (see Fig. 1). The evolution of the intra-cavity field $E$ is described by a single mean-field equation $[36,11]$ :

$$
\partial_{t} E=E_{I}-(1+i \theta) E-2 i C \rho_{13}+i \nabla^{2} E
$$

where $E_{I}$ is the amplitude of the pump field and is detuned by $\Delta$ (normalized to the atomic linewidth) from resonance of the atomic transition $|3\rangle \rightarrow|1\rangle$ while the coupling beam $E_{2}$ is kept at resonance with the transition $|3\rangle \rightarrow|2\rangle$. All fields $E, E_{I}$ and $E_{2}$ are normalized to the square root of the saturation intensity of the first atomic transition. $\theta$ is the detuning between the cavity resonance and the frequency of the injected pump beam normalized to the inverse of the photon lifetime, and $\rho_{13}$ is the off-diagonal density matrix element proportional to the field amplitude $E$ and the complex susceptibility $\chi$ via the relation $[36,37]$ :

$$
\rho_{13}=\chi E=\frac{\Delta\left|E_{2}\right|^{2}\left(\left|E_{2}\right|^{2}+|E|^{2}-i \Delta\right)}{\left(\left|E_{2}\right|^{2}+|E|^{2}\right)^{3}} E,
$$

$C$ is the co-operative parameter directly proportional to the atomic density $n_{a}$ through:

$$
2 C=\frac{n_{a} \mu^{2} k L}{2 \hbar \gamma \epsilon_{0} T},
$$


where $\mu$ is the atomic transition dipole moment, $k$ the wave number of the field, $L$ the length of the cavity, $\gamma$ the atomic linewidth, $\epsilon_{0}$ the permittivity of free space, and $T$ is the cavity mirror transmittivity. The diffraction term is given by the Laplacian operator in two transverse dimensions perpendicular to the direction of propagation. Time $t$ is normalized to the photon life time in the cavity. For convenience we fix the values of $\Delta$ to $0.2, \theta$ to -1 and $\left|E_{2}\right|^{2}$ to 1 while $2 C$ is taken as the control parameter of the system along with $E_{I}$. We note that variations in the $2 C$ parameter are possible by controlling the atomic density $n_{a}$ inside the atomic vapor cell. Eq. 1 represents the generalization of the Lugiato-Lefever two-level model $[38,39]$ to a three-level atomic configuration.

A typical experimental arrangement consistent with the theory above can be found in [40] where the experiment was carried out in a three-level $\Lambda$-type system of ${ }^{87} R b$ atoms using the $D_{1}$ lines of $5^{2} S_{1 / 2} \rightarrow 5^{2} P_{1 / 2}$ transitions. The injected beam was tuned to the atomic transition $F=1,5^{2} S_{1 / 2}$ to $F^{\prime}=2,5^{2} P_{1 / 2}$ while $E_{2}$ was set to the atomic transition $F=2,5^{2} S_{1 / 2}$ to $F^{\prime}=2,5^{2} P_{1 / 2}$. In such experiments where atomic ground states and optical fields have small frequency differences (few $\mathrm{GHz}$ ), polarizing beam splitters are used to introduce orthogonal polarizations so that the coupling beam $E_{2}$ is not oscillated in the cavity. We are considering here only electronic effects which are much faster than atomic diffusion and the onset of light forces due to intensity gradients.

We carried out a linear stability analysis of the stationary states of Eq. 1 by using an ansatz of the form $E=E_{s}+\delta E_{s} e^{\lambda t-i\left(k_{x} x+k_{y} y\right)}$, where $E_{s}, \delta E_{s}, \lambda$, and $k_{\perp}$ denote, respectively, the homogeneous stationary field, its deviation from the steady-state value, the eigenvalue corresponding to the growth rates of the ansatz and transverse wave vectors. Once the ansatz is introduced into the homogeneous stationary equation, it can be solved along with its complex conjugate to obtain a characteristic equation for the unstable spatial wave vectors. These wave vectors rule the periodicity of the incoming pattern in a mechanism typical of Turing pattern formation [41, 42]. In conservative systems like nonlinear Schrödinger equation in the presence of dispersion, similar instabilities have been called modulational instabilities. Our complex Eq. 1 is, however, dissipative and the presence of diffraction makes it mathematically equivalent to typical Turing diffusive systems as demonstrated in [42]. As the details of the global behavior of the model can be found in [11], we just recall here some specific cases of the solutions for future reference. All numerical simulations of Eq. 1 have been performed with a Fourier split-step integrator method in two spatial dimensions [11]. Initial conditions where either final configurations obtained from previous integrations or zero fields with small (less than $10^{-3} E_{s}$ ) random fluctuations (noise).

We consider parameter values above a Turing instability leading to the formation of spatially modulated structures. These structures including hexagons, rolls, honeycombs, wavy rolls, labyrinthine etc. (the latter is characterized for having a well-defined wavelength but a small correlation length thus lacking any recognizable direction which finally leads to an elaborated spatial pattern) appear in the transverse plane perpendicular to the direction of propagation. For the value of $2 C=19.60\left(\left|E_{I}\right|^{2}=\right.$ 

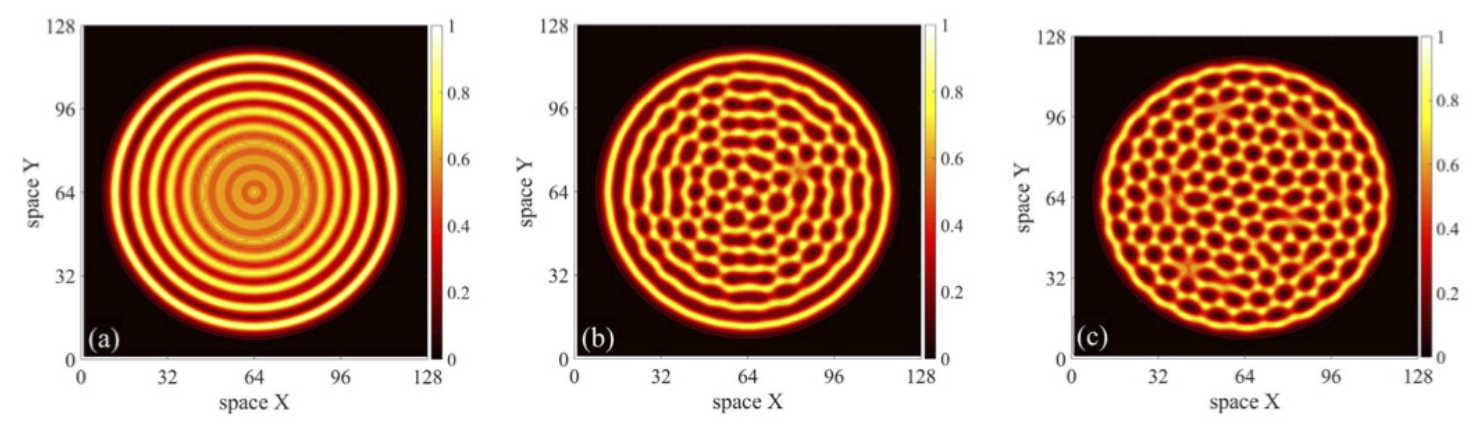

Figure 2. (Color online) A typical DOS pattern evolving from noise at $2 C=19.02$ $\left(\left|E_{I}\right|^{2}=1.201\right)$ at the beginning (a), 100 time units later (b) and the final state (c). We note that due to the circular pump shape, which removes the periodic boundary, the scrolling motion ceases but the reshaping of pattern within the finite boundary continually persists.

1.2060) branches of solutions above a Turing instability feature bistable HoneycombRoll patterns. This bistability remains present in the interval $2 C=19.55-19.75$ $\left(\left|E_{I}\right|^{2}=1.2055-1.2074\right)$. There is a small interval between stable roll and bistable Honeycomb-Roll solutions in which wavy rolls are stable. Wavy rolls loose their stability to rolls as one moves further away from the bistable solutions at higher values of $2 C$. Moreover, when the starting point of the simulations was $2 C=18.90\left(\left|E_{I}\right|^{2}=1.200\right)$ a branch which includes Distorted-Oscillating-Scrolling (DOS) honeycomb solutions is observed. In Fig. 2 a typical DOS evolution forming at $2 C=19.02\left(\left|E_{I}\right|^{2}=1.201\right)$ with circular pump shape is illustrated. These spatial structures feature patterns with orientation disorder, i. e. without any well-defined axis of symmetry in the far-field to characterize its geometrical shape, as well as a scrolling motion with no pre-assigned direction [43]. Although spatially irregular in nature, their velocity is constant in magnitude but changes with changes of the control parameter $2 C$. Local oscillation in the intensity of the DOS patterns have frequencies that are proportional to the scrolling velocity. This sort of behavior for DOS patterns has been shown to be universal and is present in disordered patterns in several types of nonlinear optical systems [43].

In our system, pattern multi-stability is found in the interval $2 C=18.75\left(\left|E_{I}\right|^{2}=\right.$ $1.2002)$ to $19.04\left(\left|E_{I}\right|^{2}=1.2017\right)$. Patterns are stationary at the beginning of the interval, but later they unlock to form scrolling structures with velocity increasing with 2C. This scrolling behavior induces local oscillations in the intensity of the pattern in a quasi-periodic way. Finally, locking takes place again and stable regular honeycomb pattern appears. Considering the fact that the preceding branch of the DOS patterns is also a stable honeycomb solution with a different wave number, one can conclude that these solutions are located at the crossing point of two basins of attraction related to the two stable honeycomb patterns with different wave-vector structures.

By considering an open-loop scheme [32] a perturbation with an amplitude appropriate for the targeted state is applied by adjusting one of the control parameters of the system, 


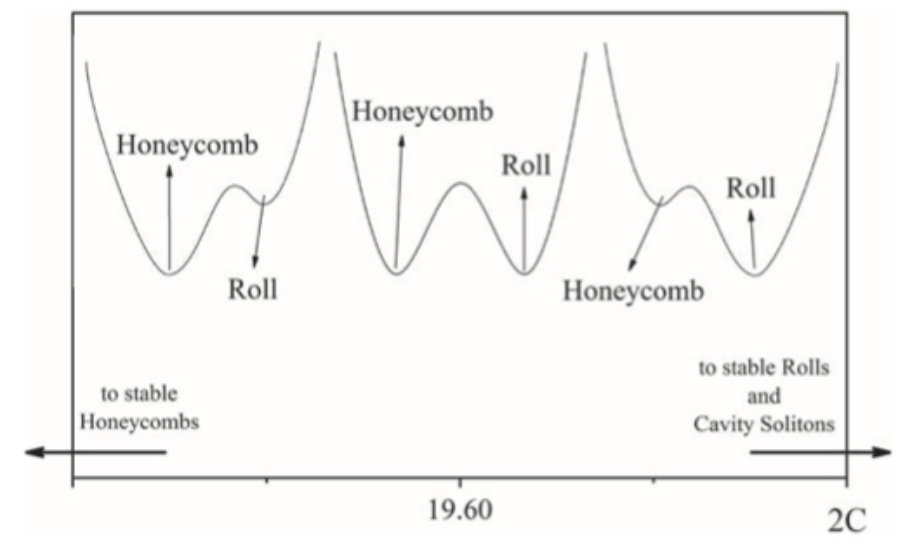

Figure 3. Schematic representation of the two competing basins of attraction in the bistable region. For higher $2 C$ values the basin associated with the roll solution is more stable while for lower $2 C$ values the honeycombs are more stable.

here $E_{I}$, and by changing a weak periodic signal in the form of a continuous sinusoidal modulation for a given time $t_{\text {pert }}$ (also normalized to the photon lifetime in the cavity). This is done in the simulations by using a function of the form:

$$
E_{I}(t)=E_{I}[1+A \sin (\omega t)] \text {. }
$$

Both the amplitude $A$ (defined as a small fraction of the injected field) and frequency $\omega$ (oscillations per time unit t.u. ${ }^{-1}$ ) of the control signal in the sinusoidal modulation have important effects in the transition and modification of the solutions. We will show that for the bistable and DOS patterns at given values of $2 C$, the value of $A$ required to reach a given target is different depending on how far one is from the desired basin of attraction.

\section{Transition of a multi-component pattern to its constituents}

In the case of patterns where both honeycomb and roll structures co-exist, bistability between these spatially periodic solutions is observed. Bifurcation points where one or the other of the patterns lose their stability are found when changing the control parameter, in our case $2 C$. In the bistable regime, the presence of two competing basins of attraction makes it possible to reach structures where boundaries between patterns are stable and even where one pattern is nested inside the other. In such cases, the spatial configuration of the field is divided into separate regions each displaying one or the other of the two stable solutions. In normal cases of no competing sets outside the bistable regime, the asymptotic spatial configuration of the field is a single regular pattern, a honeycomb structure for lower values of $2 C$ and a roll structure for larger values of $2 C$. An example of such mechanism is schematically shown in Fig. 3.

Since in the bistable region both patterns have basins of attraction, we are able to decompose the solution of coexisting patterns into its elements by a proper selection 

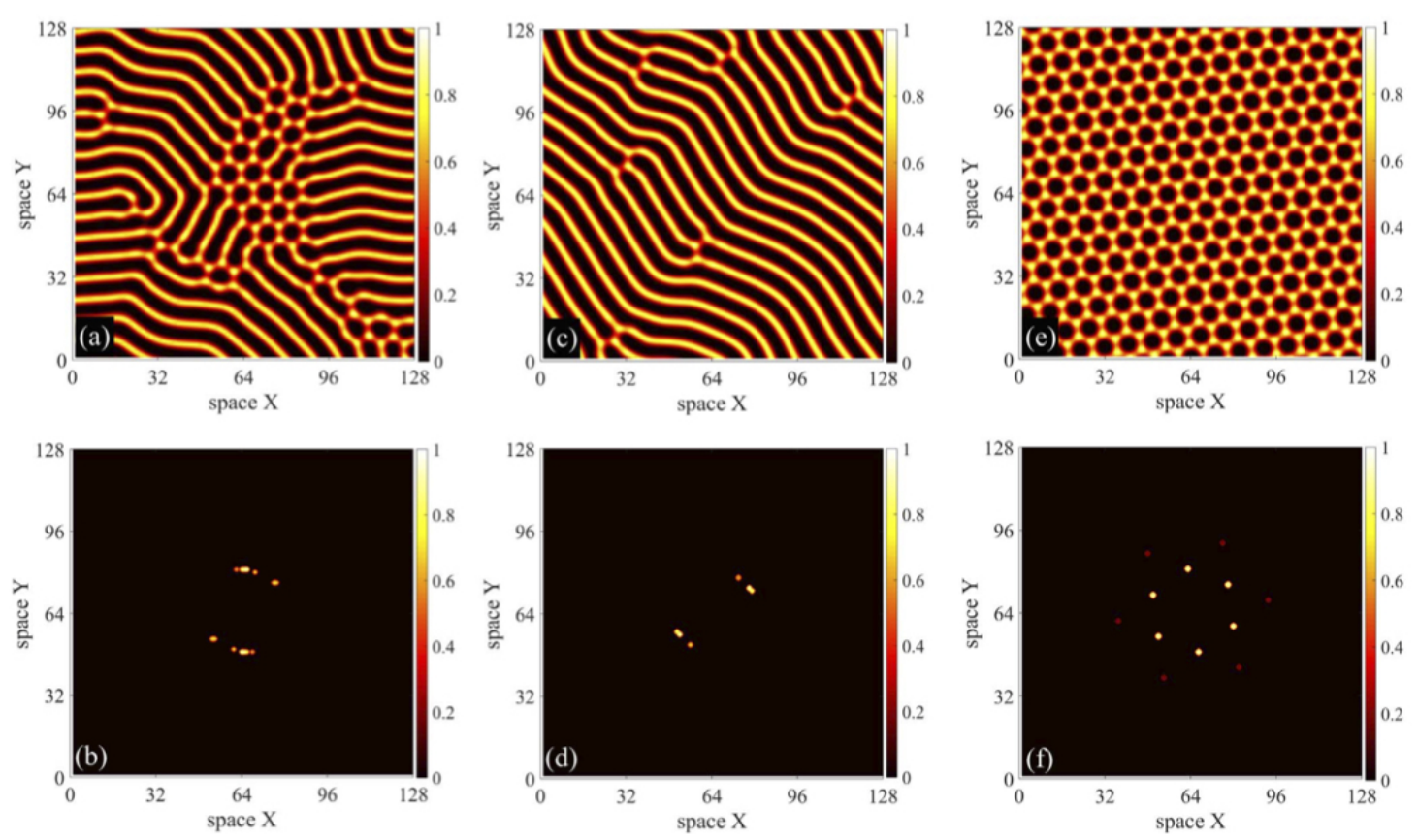

Figure 4. (Color online) Honeycomb-Roll co-existing pattern forming at $2 C=19.65$ $\left(\left|E_{I}\right|^{2}=1.2064\right)$ (a) and its far-field image (b). The wavy roll pattern obtained by applying a low amplitude harmonic perturbation (c) and the corresponding farfield image (d). Honeycomb solution from applying a higher amplitude sinusoidal perturbation (e) and the far-field image (f). The simulations are done in a $128 \times 128$ box with the size of $20 \lambda_{c}$ where $\lambda_{c}$ is the pattern wavelength.

of the amplitude of the perturbation $A$, its frequency $\omega$ and duration $t_{\text {pert }}$. For the case of coexisting patterns observed at $2 C=19.65\left(\left|E_{I}\right|^{2}=1.2064\right)$, see Fig. 4(a) and Fig. 4(b) for the near and far-field images, respectively, we have used an amplitude of $A=0.03$, a frequency of $\omega=55 \times 10^{-3}$ and a duration of $t_{\text {pert }}=5000$ to induce a stable roll and correspondingly remove the honeycomb component. Note that the forcing frequency has been chosen to be very close to that of the relaxations to the honeycomb solution. The evolution from the structure with coexisting patterns leads to a wavy roll solution (see Fig. 4(c) and (d) for the near and far-field images) during and after the application of the directing signal. The time evolution of the transition is depicted in Fig. 5(a) where the maximum of the intensity of the solution is shown along with the variations in the injected beam intensity.

On the other hand, by increasing the amplitude of the sinusoidal perturbation to $A=0.05$ but by maintaining the same frequency and duration of the control signal, we attain the stable honeycomb solution. The actual pattern and the far-field image at the end of this transition are shown in Fig. 4(e) and (f) and the related time evolutions of the solution intensity in Fig. 5(b). As implied from Fig. 3, increasing (decreasing) $2 C$ gives more stability to roll (honeycomb) solution. The situation is very similar to a double-well configuration as discussed in [44]. The application of a harmonic modulation pushes the double-well potential up and down in an asymmetrical manner 

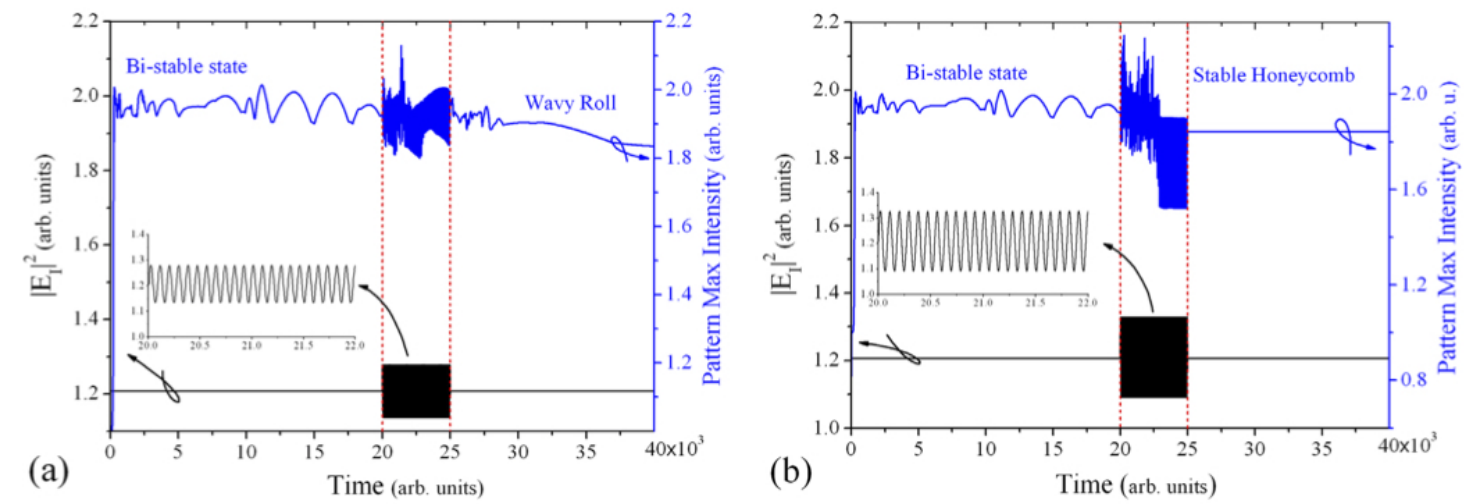

Figure 5. (Color online) Time evolution of the maximum intensity of the solution (right axis). (a) From the bistable state to the final wavy roll solution. The modulation applied on the injected beam as the control signal is on the left axis. (b) From bistable state to the final honeycomb pattern. The modulation applied on the injected beam as the control signal is on the left axis. The control was applied for 5000 time units between the two vertical dashed lines. Fluctuations in the intensities of the multistructure pattern and of the wavy roll are due to the reshaping of the structure to reach its final geometry.

and thus induces an increase and decrease of the potential barrier height in a periodic manner. If the amplitude and frequency of the applied periodic force are appropriate, the trajectory will be able to jump from one well to another. In the purely temporal case, a similar situation has been described in optical bistability in $[45,44,46]$. Therefore, to achieve the transition to either of the two solutions with different control parameter values one should consider different modulation amplitudes. Fig. 6 presents the ranges of modulation amplitudes leading to different pattern structures in the bistable regime. Two facts from Fig. 6 should be pointed out: 1) the maximum amplitude of the perturbation to reach the roll solution is almost the same as the minimum for reaching the honeycomb pattern with the exception of the case corresponding to $2 C=19.60$ for which there exists a wide gap between these values. In the gap, delimited between $A=0.018-0.05$, all the perturbations relax back to a state with coexisting patterns. ii) Transition to roll solution is not possible for the coexistent patterns for $2 C<19.60$ while one can observe a wide band of available amplitudes for a transition to honeycomb structures. Analogously, a transition to honeycomb patterns is not possible starting from coexisting patterns for $2 C>19.70$ while a wide band of available amplitudes exists for the transition to roll from a coexisting pattern at $2 C=19.75$ extends up to $A=0.05$. The physical mechanism underlying the control via the harmonic modulation signal is the annihilation of one of the existing attractors and its basin of attraction as originally described in $[45,47]$ for nonlinear oscillations in purely temporal systems described by discrete maps or ordinary differential equations. It is remarkable that the harmonic modulation method works also in our case of a spatio-temporal 


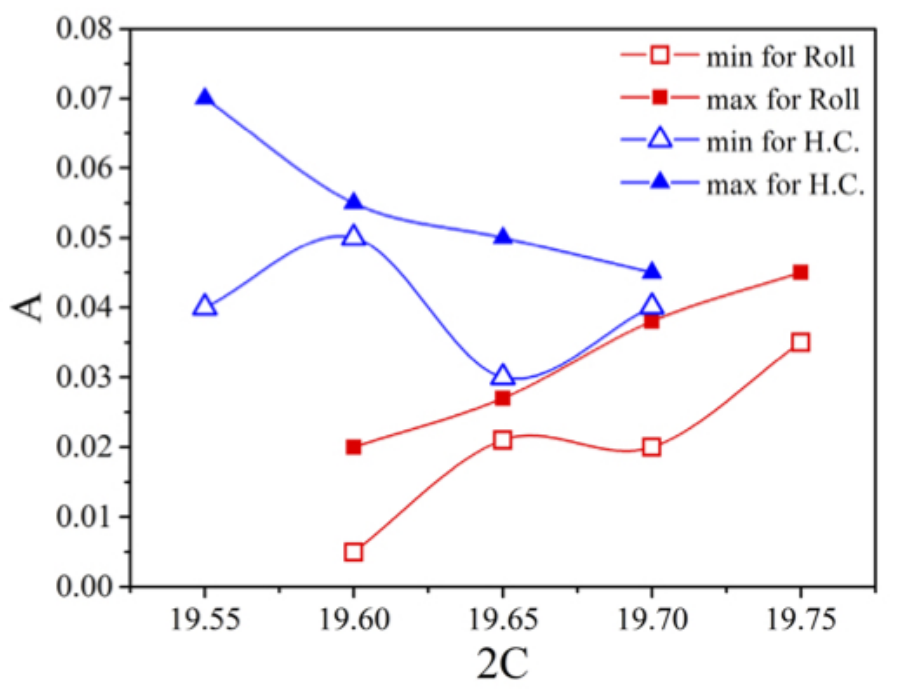

Figure 6. (Color online) Ranges of modulation amplitudes of the control signal for bistable patterns forming in different $2 \mathrm{C}$ values. H.C. stands for honeycomb.

system described by partial differential equations. The resonant interaction of the control frequency with the frequency of damped oscillations of the associated attractor results in the annihilation of one or the other attractors. Resonance peaks during modulation are observed in Fig. 5. The annihilation mechanism is evident at the beginning and at the end of Fig. 6 where the weaker attractor (rolls for lower values of $2 C$ and honeycombs for higher values of $2 C$ ) is never recovered for any amplitude of the control signal. In agreement with [30], above a pattern formation threshold there exists a large number of unstable solutions corresponding to different geometries and wave-vectors of spatially periodic structures. During modulation, one of the originally stable patterns may lose stability via a boundary crisis induced by one of the many unstable solutions. With boundary crises we mean that the related stable attractor can suddenly be destroyed when colliding with one of the many unstable solutions of the system. The situation is more complicated in the central part of Fig. 6 where different amplitudes of the control signal can lead to the annihilation of either the roll (blue lines) or honeycomb (red lines) patterns. Since the modulation frequency is close to resonance to the honeycomb annihilation, the red curves corresponding to a final roll pattern are below the blue ones for a honeycomb in the central part of Fig. 6. In this region of parameter space, the roll and honeycomb patterns have very similar stability in terms of linear eigenvalues. Then, small changes in the amplitude of the control modulation can induce either the annihilation of the honeycomb or that of the roll pattern as shown for example for the value of $2 C=19.65$ in Fig. 6. For smaller values of $2 C$, the two solutions acquire rather different eigenvalues and stabilities such that 
the amplitudes $A$ required for pattern transitions drift apart and leave a gap where transition to neither of the solutions is possible, see for example $2 C=19.60$ in Fig. 6 .

\section{Decomposition of distorted patterns and removal of defects}

A consequence of sensitivity of the spatio-temproal solutions to initial conditions is the presence of stable defects when the initial value of the control parameter is around $2 C=18.90$. The behavior of irregular structures and DOS patterns, are detailed in [11] in terms of the local defects, periodicity of the oscillations and their scrolling motion. Removal of defects from disordered patterns in nonlinear optics has been achieved in [35] by adding an all-optical feedback loop with a spatial Fourier filter blocking the discrete number of modes which constitute the target pattern. In that scheme, the remaining control signal was then negatively fed back into the system driving it towards the target state. Although special in providing active manipulation, this approach acts on the defected regions gradually and individually avoiding any sudden change in the entire structure. Here we use a technique based on a harmonic perturbation of finite duration that selects one of the coexistent solutions and drives the system to the selected stable pattern at once with advantages in the simplicity of operation.

A spatial structure with defects can be controlled by transferring the dynamics to the stable attractors nearby, which are stable honeycombs with different wave-numbers. Similar to the case of Honeycomb-Roll bistable pattern involving two competing basins of attraction with different characteristics, a proper choice of amplitude $A$, frequency $\omega$ and duration $t_{\text {pert }}$ of the control signal for the specific case of DOS pattern is capable to induce a transition to one of the stable honeycomb solutions that existed before or after the DOS interval. In this way, one has the possibility of choosing between two honeycomb solutions with slightly different wave numbers. This can be seen by noting that the DOS structures are delimited between two stable honeycomb branches with different wave-vector structures. For a typical transition away from the DOS structure of Fig. 7(a) and (b) to stable honeycomb solution shown in Fig. 7(c) and (d), we have chosen the amplitude of $A=0.04$, frequency of $\omega=42 \times 10^{-3} t \cdot u .^{-1}$ and duration $t_{\text {pert }}=10000$.

Higher amplitudes of the modulation, for example $A=0.05$, but the same frequency of modulation, move the system to a second attractor possible as shown in Figs. 7(e) and (f). It is interesting to note from Figs. 7(d) and (f) that the final stable honeycomb patterns differ from each other in orientation and wave-number (respectively 0.858 and 0.844 ). These two wave-vectors coexist in the original DOS structure displayed in Figs. 7(a) and (b). This is consistent with the general definition of defects formed by the simultaneous presence of two attracting sets for a specific pattern differing by wave-numbers [13]. Therefore, removal of defects from the original structure is, in fact, nothing but a decomposition into the involved spatial modes. The corresponding intensities in the transition from DOS to stable honeycomb solutions along with the holding 

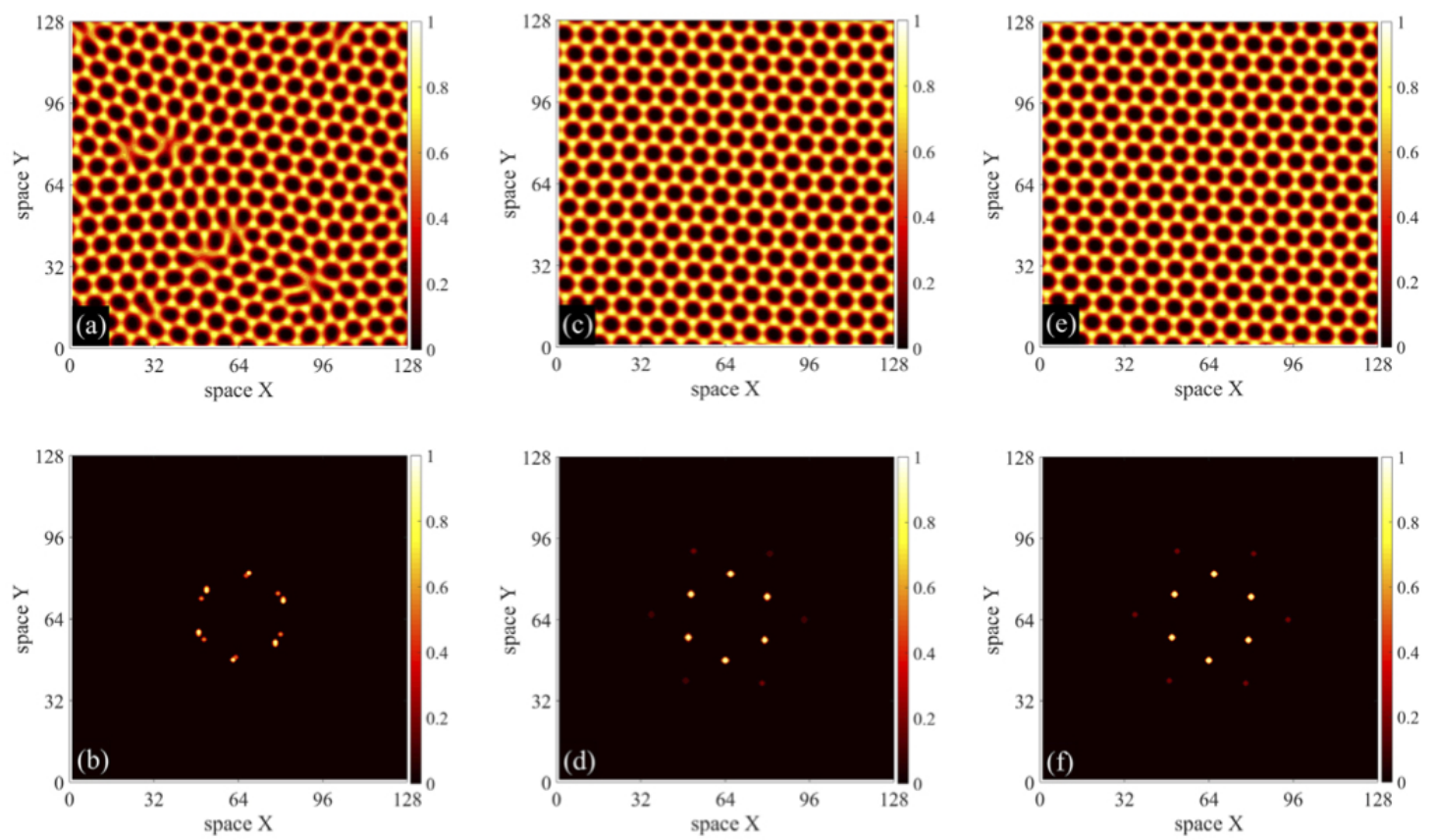

Figure 7. (Color online) Defected honeycomb solution forming at 2C=19.02 (a) and its far-field image (b). Stable honeycomb solutions obtained from applying a harmonic perturbation of amplitude $A=0.04$ (c) and $A=0.05$ (e) along with their far-field images in (d) and (f). The simulations are performed in a $128 \times 128$ grid with $20 \lambda_{c}$ of size.

beam variations are shown in Fig. 8 for the two different perturbation amplitudes. To further demonstrate that the two final stable honeycombs are indeed different solutions, we have provided their phase evolutions in Fig. 9.

\section{The control mechanism in the case of a finite pump}

In order to guarantee that the control method introduced above is not affected by the periodic boundary conditions used in the simulations, we have replaced the flat pump with one of finite size and circular boundary closer to real experimental conditions. A flat-top injection with rapidly vanishing tails is introduced to the simulations through:

$$
P_{I}(r)=\frac{E_{I}}{2}\left\{1-\tanh \left[\sigma\left(r-r_{0}\right)\right]\right\}
$$

where $\sigma$ and $r_{0}$ regulates the size of the tail and flat part of the pump, respectively. $P_{I}(r)$ replaces $E_{I}$ in Eq. 1. In Fig. 10(a) a structure formed by the two bistable patterns separated by a front is displayed. By applying the control technique introduced in Sec. III with $A=0.04, \omega=42 \times 10^{-3} t . u .^{-1}$ and $t_{\text {perp }}=10000$, a transition to a roll solution is observed as shown in Fig. 10(b). By changing $A$ to $A=0.085, \omega$ to $\omega=98 \times 10^{-3}$ t.u. ${ }^{-1}$, and $t_{\text {perp }}$ to $t_{\text {perp }}=5000$ a transition to a honeycomb solution is obtained as shown in Fig. 10(c). Note that the circular pump deforms the geometry of the honeycomb cells 

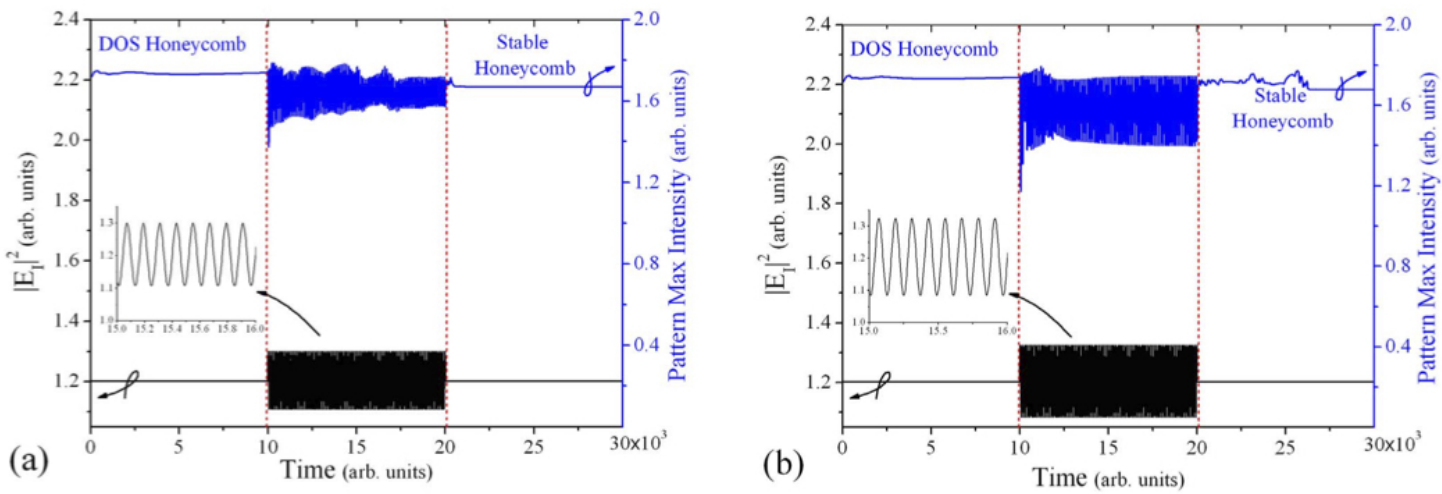

Figure 8. (Color online) Intensities associated to the solutions; from the defected honeycomb state to the stable honeycomb solution (right axis) and the modulation applied on the holding beam as the control (left axis). The amplitudes of control were $A=0.04$ and $A=0.05$ respectively for (a) and (b) while the frequency was the same for both $\left(\omega=42 \times 10^{-3} t . u .^{-1}\right)$. The control was on for $t_{\text {pert }}=10 \times 10^{3}$ time units and depicted by two vertical dashed lines. Fluctuations seen in the intensity of the initial state is due to the slight scrolling of the defected pattern.

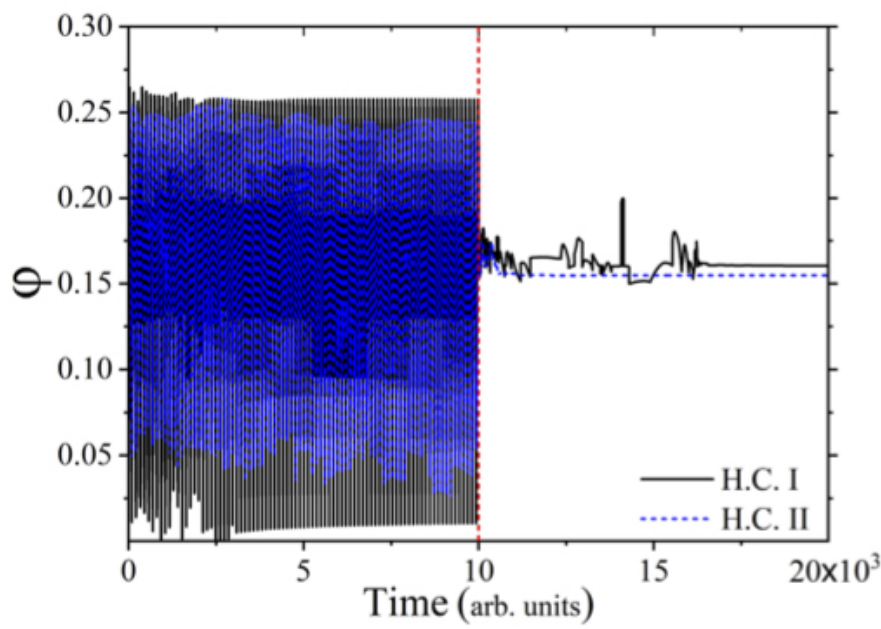

Figure 9. (Color online) Phase evolution of the output for the two modulation amplitudes ( $A=0.04$ and $A=0.05$ respectively for solid and dashed lines). It is clearly seen that the initial solution is transferred to two honeycombs differing by their phase (0.154 and 0.161$)$ in addition to orientation and wave-numbers. Note that the phase status of the initial DOS pattern is irregular in time and not shown here. The dashed vertical line marks the end of modulation and the values are the same as Fig. 8. 

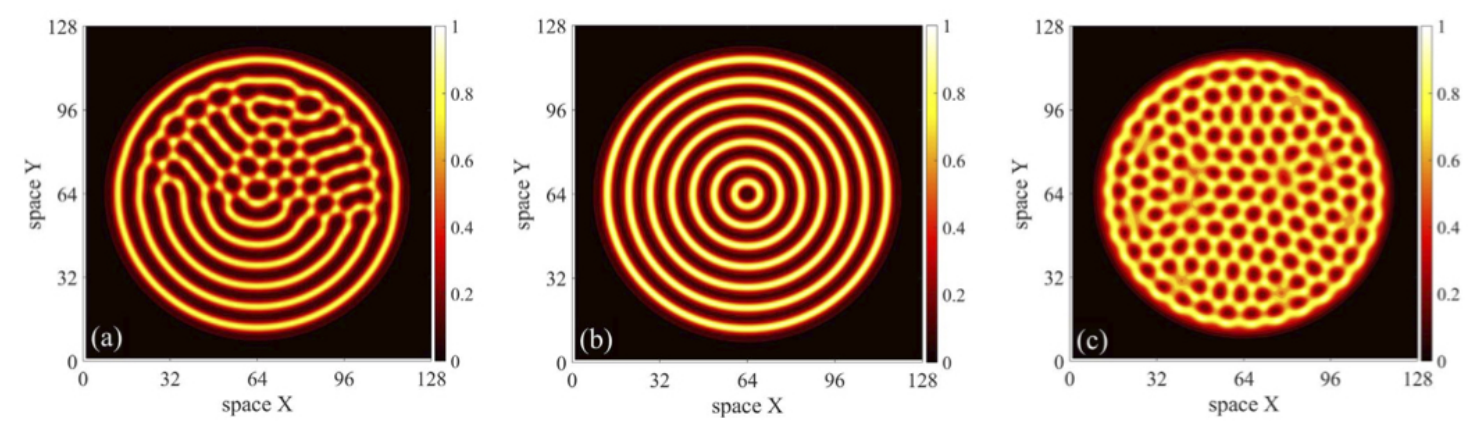

Figure 10. Honeycomb-Roll bistable pattern in presence of finite pump (a), the final structure from transition to rolls with $A=0.04, \omega=42 \times 10^{-3} t . u .^{-1}$, and $t_{\text {perp }}=10000$ (b) and the one of honeycomb at the end of modulation with $A=0.085$, $\omega=98 \times 10^{-3} t . u .^{-1}$, and $t_{p e r p}=5000(\mathrm{c})$. For (a) and (b) we have used the values $2 C=19.55, \sigma=10 / \lambda_{c}$ and $r_{0}=9 \lambda_{c}$, while for (c) we had to use a lower value of $2 C=19.45$ to ensure the stability of honeycomb solution since presence of circular injection gives more stability for the roll solution by moving the system towards the basin related to roll structures.

as they tend to be perpendicular to the boundary. The harmonic perturbation method appears to be robust with respect to finite size spatial structures. If one removes the circular boundary after the generation of the structure displayed in Fig. 10(c), a regular honeycomb pattern is fully recovered. The harmonic perturbation method has also been successfully applied to the case of removal of defects with finite size input beams.

\section{Conclusions}

Pattern multi-stability and sensitivity to initial conditions are generic features of diffraction in a cavity with a medium close to EIT. A mechanism to select single one of the simultaneously present stable solutions and based on the harmonic perturbation of the input pump has been proposed and tested in numerical simulations. In particular, we have shown that by selecting appropriate amplitudes, frequencies and duration of the control signal, one can induce transitions of structures formed by coexisting patterns into either of their fundamental components. Simulations show that amplitude of the perturbation is the key to select the desired solution while frequency can be kept fixed to values close to the characteristic decay rates to the stationary patterns in the optical cavity. The control method based on harmonic signals works by changing the stability of one or more of the originally multi-stable states. Critical values of the amplitude of the control signal then drive the dynamical trajectory of the system towards one of the remaining attractors. We have extended this technique to a spatio-temporal system with an infinitely large phase space to attain a roll or a honeycomb pattern starting from configurations that contained domains of both. We believe that the same technique can be generalized to the case of competition among three or more patterns rather than two pattern 
structures discussed in this paper. We have also discussed the extension of the proposed control mechanism to remove defects from distorted patterns when two solutions of the same kind but with different wave-vector structures are simultaneously present. This gives the possibility of removing the defects by transferring the irregular state to one of the stable solutions with a particular wave-number. Physical relevance of the proposed control mechanism has been verified by using a pump with flat-top shape, finite size and vanishing tails to avoid any consequences of periodic boundary conditions used in the simulations.

\section{Acknowledgment}

M. E. acknowledges the support by Iran National Science Foundation (INSF) under the grant No. 95008093. 
Complex structures in cavities with media displaying EIT...

\section{References}

[1] M. Fleischhauer, A. Imamoglu, and J. P. Marangos, Rev. Mod. Phys. 77, 633 (2005).

[2] L. V. Hau, S. E. Harris, Z. Dutton, and C. H. Behroozi, Nature 397, 594 (1999).

[3] D. Budker, D. F. Kimball, S. M. Rochester, and V. V. Yashchuk, Phys. Rev. Lett. 83, 1767 (1999).

[4] D. F. Phillips, A. Fleischhauer, A. Mair, R. L. Walsworth, and M. D. Lukin, Phys. Rev. Lett. 86, 783 (2001).

[5] D. Budker, W. Gawlik, D. F. Kimball, S. M. Rochester, V. V. Yashchuk, and A. Weis, Rev. Mod. Phys. 74, 1153 (2002).

[6] V. M. Acosta, K. Jensen, C. Santori, D. Budker, and R. G. Beausoleil, Phys. Rev. Lett. 110, 213605 (2013).

[7] A. S. Zibrov, M. D. Lukin, D. E. Nikonov, L. Hollberg, M. O. Scully, V. L. Velichansky, and H. G. Robinson, Phys. Rev. Lett. 75, 1499 (1995).

[8] M. C. Phillips,H. Wang, I. Rumyantsev, N. H. Kwong, R. Takayama, and R. Binder, Phys. Rev. Lett. 91, 183602 (2003).

[9] D. Schraft, M. Hain, N. Lorenz, and T. Halfmann, Phys. Rev. Lett. 116, 073602 (2016).

[10] H. Q. Fan, K. H. Kagalwala, S. V. Polyakov, A. L. Migdall, and E. A. Goldschmidt, Phys. Rev. Lett. 99, 053821 (2019).

[11] M. Eslami, R. Kheradmand, D. McArthur, and G.-L. Oppo, Phys. Rev. A 90, 023840 (2014).

[12] For an example outside optics see A. Yochelis, Y. Tintut, L. L. Demer, and A. Garfinkel, New J. Phys. 10, 055002 (2008).

[13] D. Walgraef, Spatio-Temporal Pattern Formation, (Springer-Verlag, Berlin, 1997).

[14] M. Eslami, M. Khanmohammadi, R. Kheradmand, and G.-L. Oppo, Phys. Rev. A 96, 033836 (2017).

[15] F. T. Arecchi, R. Meucci, G. P.. Puccioni, and J. R. Tredicce, Phys. Rev. Lett. 49, 1217 (1982).

[16] A. N. Pisarchik, Y. O. Barmenkov, and A. V. Kir'yanov. Phys. Rev. E 68, 066211 (2003).

[17] F. Wolf, Phys. Rev. Lett. 95, 208701 (2005); J. A. S. Kelso, Phil. Trans. R. Soc. B 367, 906 (2012).

[18] C. N. Ngonghala, U. Feudel, and K. Showalter, Phys. Rev. E 83, 056206 (2011).

[19] A. Robinson, R. Calov, and A. Ganopolski, Nat. Clim. Change 2, 429 (2012).

[20] B. E. Martinez-Zerega, A. N. Pisarchik, and L. S. Tsimring. Phys. Lett. A 318, 102 (2003).

[21] M. D. Marco, M. Forti, M. Grazzini, and L. Pancioni, Neural Netw. 54, 38 (2014).

[22] M. Cross and H. Greenside, Pattern Formation and Dynamics in Nonequilibrium Systems (Cambridge University Press, Cambridge, 2009).

[23] L. A. Lugiato, G.-L. Oppo, J. R. Tredicce, L. M. Narducci, and M. A. Pernigo, J. Opt. Soc. Am. B 7, 1019 (1990).

[24] M. A. Vorontsov and W. J. Firth, Phys. Rev. A 49, 2891 (1994).

[25] M. Schwab, C. Denz, and M. Saffman, Appl. Phys. B 69, 429 (1999).

[26] B. Hasselblatt and K. Anatole, A First Course in Dynamics: With a Panorama of Recent developments, (Cambridge University Press, Cambridge, 2003).

[27] S. Boccaletti, C. Grebogi, Y. C. Lai, H. Mancini, and D. Maza, Phys. Rep. 329, 103 (2000).

[28] E. Ott, C. Grebogi, J. A. Yorke, Phys. Rev. Lett. 64, 1196 (1990).

[29] J. E. S. Socolar, D. W. Sukow, and D. J. Gauthier, Phys. Rev. E 50, 3245 (1994); H. Gang and Q. Zhilin, Phys. Rev. Lett. 72, 68 (1994); S. Bielawski, D. Derozier, and P. Glorieux, Phys. Rev. E 49, R971(R) (1994); R. Meucci, W. Gadomski, M. Ciofini, and F. T. Arecchi, Phys. Rev. E 49, R2528(R) (1994); K. Pyragas, Phys. Rev. Lett. 86, 2265 (2001); P. Hövel and E. Schöll, Phys. Rev. Lett. 72, 046203 (2005).

[30] R. Martin, A. J. Scroggie, G.-L. Oppo, and W.J. Firth, Phys. Rev. Lett. 77, 4007 (1996).

[31] G. K. Harkness, G.-L. Oppo, R. Martin, A. J. Scroggie, and W. J. Firth, Phys. Rev. A 58, 2577 (1998).

[32] L. Pastur, L. Gostiaux, U. Bortolozzo, S. Boccaletti, and P. L. Ramazza, Phys. Rev. Lett. 93, 063902 (2004); L. Illing, D. J. Gauthier and R. Roy, Adv. At., Mol., Opt. Phys, 54, 615 (2007). 
[33] A. N. Pisarchik and U. Feudel, Phys. Rep. 540, 167-218 (2014).

[34] R. Sevilla-Escoboza, A. N. Pisarchik, R. Jaimes-Reátegui and G. Huerta-Cuellar, Proc. R. Soc. A 471, 20150005 (2015).

[35] R. Neubecker, E. Benkler, R. Martin, and G.-L. Oppo, Phys. Rev. Lett. 91, 113903 (2003).

[36] G.-L. Oppo, Journal of Modern Optics 57, 1408 (2010).

[37] A. S. Manka, H. M. Doss, L. M. Narducci, P. Ru, and G.-L. Oppo, Phys. Rev. A 43, 3748 (1991).

[38] L. A. Lugiato and R. Lefever, Phys. Rev. Lett. 58, 2209 (1987).

[39] L. A. Lugiato and C. Oldano, Phys. Rev. A 37, 3896 (1988).

[40] A. Joshi, A. Brown, H. Wang and M. Xiao, Phys. Rev. A 67, 041801(R) (2003).

[41] M. C. Cross and P. C. Hohenberg, Rev. Mod. Phys. 65, 851 (1993).

[42] G.-L. Oppo, J. Math. Chem. 45, 95 (2009).

[43] A. J. Scroggie, D. Gomila, W. J. Firth, and G.-L. Oppo, Appl. Phys. B 81, 963 (2005).

[44] A. Joshi and M. Xiao, Controlling Steady-state and Dynamical Properties of Atomic Optical Bistability, (World Scientific Publishing, Singapore, 2012).

[45] A. N. Pisarchik, and B. K. Goswami, Phys. Rev. Lett. 84, 1423 (2000).

[46] A. Joshi and M. Xiao, Journal of Modern Optics 57, 1196 (2010).

[47] A. N. Pisarchik, Phys. Rev. E 64, 046203 (2001). 